\title{
The physician's estimation 'alternative diagnosis is less likely than pulmonary embolism' in the Wells rule is dependent on the presence of other required items
}

\author{
Frederikus A. Klok, Majida Zidane, Reza Karami Djurabi, Mathilde Nijkeuter, Menno V. Huisman \\ Section of Vascular Medicine, Department of General Internal Medicine - Endocrinology, Leiden University Medical Center, Leiden, The \\ Netherlands
}

\section{Dear Sir,}

Since the last decade, several clinical decision rules (CDRs) in the diagnostic process of pulmonary embolism (PE) have been proposed and validated (1-4). No rule has optimal sensitivity or specificity. It is for this reason that CDRs only have efficient clinical utility as a component of diagnostic strategies in combination with other simple bedside tests as D-dimer measurement (5-8). Indeed, in case of suspected PE, the combination of a low or intermediate (7) (in case of a trichotomised outcome) or less likely (5) (in case of dichotomised outcome) clinical probability with normal D-dimer tests safely rule out PE and oral anticoagulation therapy can safely be withheld in these patients.

All CDRs have practical limitations. One of the most validated and used CDR's is the Wells rule (2). This rule includes the attending physician's judgment of whether an alternative diagnosis is less or more likely than PE. If the physician is of the opinion that PE is the most likely diagnosis, he will add an extra 3 points to the total score (Table 1). If an alternative diagnosis is as likely as or more likely than PE, he will add zero points to the total score. The subjective character of this specific criterion and its moderate reproducibility due to inter observer variability (9) is the main point of criticism to the Wells rule. Furthermore, the

Correspondence to:

M.V. Huisman

LUMC (C4-70)

Albinusdreef 2, Postbus 9600

2300 RC Leiden, The Netherlands

Tel.: +3I 7I 5262085, Fax: +3I 7I 5248I40

E-mail: M.V.Huisman@LUMC.nl

Received September 12, 2007

Accepted after minor revision November 7, 2007

Prepublished online December 5, 2007

doi: 10.II60/TH07-09-0560

Thromb Haemost 2008; 99: 244-245 presence of any alternative diagnosis which is at least as likely as $\mathrm{PE}$ reduces the likelihood of $\mathrm{PE}$, but this effect is not large enough to allow ruling out PE without further testing (10). On the other hand, this criterion enables the physician to use his medical intuition. In addition, implicit clinical judgment has been shown to improve the accuracy of another CDR (11). Finally, it was recently shown that this subjective criterion has a high predictive value in comparison to the other variables of the Wells

\section{Table I: The Wells rule.}

\begin{tabular}{|l|l|}
\hline $\begin{array}{l}\text { Variable } \\
\begin{array}{l}\text { Clinical signs and symptoms of DVT (minimum of leg swelling } \\
\text { and pain with palpation of deep veins) }\end{array}\end{array}$ & Points \\
\hline An alternative diagnosis is less likely than PE & 3 \\
\hline Heart rate greater than I00 & 1.5 \\
\hline Immobilisation or surgery in the previous four weeks & 1.5 \\
\hline Previous DVT / PE & 1.5 \\
\hline Haemoptysis & 1 \\
\hline Malignancy (on treatment, treated in the last 6 months or & 1 \\
\hline palliative) & \\
\hline Clinical probability & $<2$ total \\
\hline Low & $2-6$ total \\
\hline Intermediate & $>6$ total \\
\hline High & $\leq 4$ total \\
\hline Dichotomised assessment & $>4$ total \\
\hline Less likely & \multicolumn{2}{|l}{} \\
\hline Likely & \\
\hline DVT, deep vein thrombosis; PE, pulmonary embolism. & \\
\hline
\end{tabular}


rule and has even more diagnostic value if it plays a decisive role in whether a patient is designated likely or unlikely (12).

We hypothesised that the physician's decision to assign the 3 points for the item 'alternative diagnosis is less likely than PE' is critically or significantly influenced by the presence of one or more other variables in the Wells rule.

In order to test this, we analysed the data of a large multicenter prospective diagnostic study (5). In this study, the diagnostic strategy with the combination of dichotomised Wells rule and D-dimer tests was prospectively studied. A total of 3,306 consecutive in- and outpatients with suspected PE were included. In all patients, the Wells rule was calculated by the attending physician. We calculated an adjusted Wells rule without the 3 points for the subjective criterion. We compared the adjusted Wells rule in all patients in which the subjective criterion was awarded to all patients with another diagnosis more likely than PE, using a Mann-Whitney U test. Also, we used a logistic regression model to calculate the risk for granting the 3 points in all different totals of the adjusted Wells rule.

In 1,274 patients (39\%) an alternative diagnosis was considered to be more likely than PE (group A). In 2,032 patients
(61\%) PE was considered to be the most likely diagnosis (group $\mathrm{B}$ ). The median of the adjusted Wells rule in group A was 1.0, the median of group B was $1.5,(\mathrm{p}<0.001)$. We calculated that with each point increase in adjusted Wells rule, patients had an odds ratio of $1.2(95 \%$ CI $1.1-1.3, p<0.001)$ of being awarded with the 3 points for PE as most likely diagnosis. In conclusion, with every additional point in the adjusted Wells rule, patients had a significant 1.2-fold increased chance of being assigned the subjective criterion.

Our data show that a physician, when deciding on awarding the 3 points for most likely diagnosis, is influenced by the other variables in the Wells rule. We explain our findings from the fact that besides haemoptysis and heart rate, all other variables are well established risk factors for PE. A fully standardised rule, the revised Geneva score, has been developed and validated recently $(4,13)$. Whether the use of this score will result in a higher predictive accuracy than the use of the Wells rule should be studied prospectively by directly comparing these two decision rules in outcome studies.

\section{References}

1. Wells PS, Ginsberg JS, Anderson DR, et al. Use of a clinical model for safe management of patients with suspected pulmonary embolism. Ann Intern Med 1998; 129: 997-1005.

2. Wells PS, Anderson DR, Rodgers M, et al. derivation of a simple clinical model to categorize patients probability of pulmonary embolism: increasing the models utility with simpliRED D-dimer. Thromb Haemost 2000; 83: 416-420.

3. Wicki J, Perneger TV, Junod AF, et al. Assessing clinical probability of pulmonary embolism in the emergency ward. Arch Intern Med 2001; 161: 92-97.

4. Le Gal G, Righini M, Roy P-M, et al. Prediction of pulmonary embolism in the emergency department: the revised Geneva score. Ann Intern Med 2006; 144: 165-171.

5. van Belle A, Büller HR, Huisman MV, et al.; Christopher Study Investigators. Effectiveness of managing suspected pulmonary embolism using an algorithm combining clinical probability, D-dimer testing, and computed tomography. J Am Med Assoc 2006; 295 : $172-179$.

6. Kruip MJ, Slob MJ, Schijen JH, et al. Use of a clinical decision rule in combination with $\mathrm{D}$-dimer concentration in diagnostic workup of patients with suspected pulmonary embolism: a prospective management study. Arch Intern Med 2002; 162: 1631-1635.

7. Leclercq MG, Lutisan JG, van Marwijk Kooy M, et al. Ruling out clinically suspected pulmonary embolism by assessment of clinical probability and D-dimer levels: a management study. Thromb Haemost 2003; 89: 97-103.

8. Goekoop RJ, Steeghs N, Niessen RW, et al. Simple and safe exclusion of pulmonary embolism in outpatients using quantitative D-dimer and Wells' simplified decision rule. Thromb Haemost 2007; 97: 146-150

9. Leclerq MGL, Kruip MJHA, Mac Gillavry MR, et al. Observer variability in the assessment of clinical probability in patients with suspected pulmonary embolism. J Thromb Haemost 2004; 2: 1204-1206.

10. Testuz A, Le Gal G, Righini M, et al. Influence of specific alternative diagnoses on the probability of pulmonary embolism. Thromb Haemost. 2006; 95: 958-962.

11. Chagnon I, Bounameaux H, Aujesky D, et al. Comparison of two clinical prediction rules and implicit assessment among patients with suspected pulmonary embolism. Am J Med 2002; 113: 269-275.

12. Klok FA, Karami Djurabi R, Nijkeuter M, et al. Alternative diagnosis other than pulmonary embolism as a subjective variable in Wells clinical decision rule; not so bad after all. J Thromb Haemost 2007; 5: 1079-1080.

13. Klok FA, Kruijswijk E, Spaan J, et al. Comparison of the revised Geneva score with the Wells rule for assessing clinical probability of pulmonary embolism. J Thromb Haemost 2007; Oct 20 [Epub ahead of print] 